\title{
Nanocurcumin alleviate inflammation and oxidative stress in LPS-induced mastitis model via activation of Nrf2 and suppressing TLR4 mediated NF-KB and HMGB1 signaling pathways
}

Mohamed A. Lebda ( $\sim$ lebdam1979@alexu.edu.eg )

Department of Biochemistry Faculty of Veterinary Medicine Alexandria University

Ingi $\mathrm{H}$. Elmassry

Alexandria University Faculty of Veterinary Medicine

Nabil M. Taha

Alexandria University Faculty of Veterinary Medicine

Mohamed S. Elfeky

Alexandria University Faculty of Veterinary Medicine

\section{Research Article}

Keywords: Mastitis, LPS, nanocurcumin, TLR4, Oxidative stress

Posted Date: May 11th, 2021

DOl: https://doi.org/10.21203/rs.3.rs-447345/v1

License: (c) (1) This work is licensed under a Creative Commons Attribution 4.0 International License.

Read Full License 


\section{Abstract}

Mastitis is a worldwide serious disease of the mammary gland. Curcumin is a pleiotropic polyphenol obtained from turmeric, but it is hydrophobic and rapidly eliminated from the body. However, nanoformulation of curcumin significantly improves its pharmacological activity by enhancing its hydrophobicity and oral bioavailability. Our study aimed to investigate the possible anti-oxidant and antiinflammatory effects of nanocurcumin as a prophylactic against LPS-induced mastitis in rat model; where LPS was extracted from a field strain of Escherichia coli (bovine mastitis isolate). The study was conducted on twenty lactating Wistar female rats divided into four equal groups and the mastitis model was initiated by injection of LPS through the duct of the mammary gland. The results showed that nanocurcumin significantly attenuated the lipid peroxidation (MDA), oxidized glutathione, the release of pro-inflammatory cytokines (TNF- $\alpha$ and IL-1 $\beta$ ) as well as the gene expression of TLR4, NF-kB p65, and HMGB1. Meanwhile, it improved the reduced glutathione level, Nrf2 activity and preserved the normal alveolar architecture. These findings suggested that nanocurcumin supplementation can be a promising potential protective approach for coliform mastitis.

\section{Introduction}

Mastitis is a global inflammatory disease of the mammary gland (MG) associated with detrimental economic losses concerning the dairy industry (Gomes \&Henriques 2016), which exceed 2 billion \$ annually according to the National Mastitis Council (Cobirka et al. 2020). These losses are due to the drop in milk production, the rise of culling rate, as well as treatment, veterinary supervision, and labor costs, accounting for $78 \%, 14 \%$, and $18 \%$ respectively; additionally to, declined conception rates and high pregnancy losses (Smulski et al. 2020). The International Dairy Federation identified that mastitis could be caused by either traumatic or toxic agents or mostly by more than 150 species of contagious and environmental infectious agents (Kibebew 2017). More than $40 \%$ of mastitis cases are caused by gramnegative bacteria, mostly coliforms (Quesnell et al. 2012). Escherichia coli (E. coli) is the most frequent species isolated, constituting nearly $80 \%$ of the intra-mammary coliform infections (Fahim et al. 2019a), with over 700 genotypically and phenotypically different serotypes (Jang et al. 2017).

Lipopolysaccharides (LPS) is a heat-stable amphiphilic molecule that represents the major constituent of the outer membrane of gram-negative bacteria, known as bacterial endotoxin, and has been reported to be responsible for the severe inflammatory reaction (Mazgaeen \&Gurung 2020). It consists of three regions: a lipophilic portion (lipid $A$ ) which is responsible for bacterial virulence and toxicity, a hydrophilic oligosaccharide, and an O- antigen (Sampath 2018). It is believed that LPS is released after bacterial death or during bacterial multiplication (Suojala et al. 2013). Thus, LPS inoculation through the milk duct mimics the symptoms of gram-negative mastitis by disrupting the permeability of the blood milk barrier (Jiang et al. 2020, Kan et al. 2019). Therefore, LPS is considered as a representative model to study mastitis caused by E.coli and its enterotoxins including the host response to inflammatory and oxidative changes without the risks resulting from a bacterial infection (Glynn et al. 2014, Jiang et al. 2020). LPS acts as a pathogen-associated pattern receptor to activate toll-like receptor 4 (TLR4) resulting in the 
initiation of the inflammatory response through downstream signaling involving the activation of Nuclear factor-kappa B (NF-KB)(Ingman et al. 2014, Khan et al. 2020), which provoke the transcription of proinflammatory cytokines including tumor necrosis factor-alpha (TNFa), interleukin (IL)1, 2, 6, 8, as well as the activation of damage-associated molecular patterns (DAMPs); like high-mobility group box-1 (HMGB1), additionally to the initiation of oxidative stress within the MG tissue (Shi et al. 2016).

Nowadays, the treatment regimen of mastitis commonly includes broad-spectrum antibiotics and nonsteroidal anti-inflammatory drugs (NSAIDs) (Suojala et al. 2013). Hence, mastitis represents a public health hazard not only due to the pathological changes of milk (Sayed et al. 2014), but also, the risk of the alimentary transmission of antibiotic resistance genes to human, as well as other demerits of NSAIDs food residues such as hepatotoxicity, diarrhea, and CNS depression (Barreto et al. 2019). Likewise, vaccination showed low efficacy in controlling mastitis due to the wide etiological range (Cheng \&Han 2020). Therefore, the application of phytochemicals has been regarded as a promising alternative prophylactic for mastitis, importantly, curcumin (Suresh et al. 2018).

Curcumin is derived from the rhizomes of Turmeric and responsible for its yellow color (Gupta et al. 2012). It is extensively cultivated in Asian countries especially India and China and was applied around 5000 years ago in traditional medicine (Rahimi et al. 2016, Rai et al. 2015). It possesses three functional groups; two phenolic groups in the form of 0 - methoxyphenyl groups attached to aromatic rings and a diketone moiety in the form of $a, \beta$ unsaturated $\beta$ - diketone (Priyadarsini 2014). Thus, it is capable to modulate several signaling molecules as well as DNA and RNA, responsible for multiple activities due to its pleiotropic property, making it efficient as an anti-inflammatory, anti-oxidant, chemoprotective, antiparasitic, and wound healing agent (Gupta et al. 2012).

Curcumin modulates the inflammatory response during mastitis by downregulating the activity of TLR4/MYD88/ NF-KB cascade (Catanzaro et al. 2018) and the suppression of the inflammatory mediators such as TNF- $\alpha$, IL-1 $\beta$ (Chen et al. 2018), and HMGB1(Wang et al. 2012). Furthermore, curcumin is a potent anti-oxidant through scavenging free radicals (Pu et al. 2014), the repression of reactive oxygen species (ROS) (de Almeida Alvarenga et al. 2018) and malondialdehyde (MDA) production, as well as the maintenance of GSH level, nuclear factor erythroid 2-related factor 2 (Nrf2) activity, and the upregulation of antioxidant enzymes (Boyanapalli et al. 2014).

Pharmacokinetic studies identified that the application of curcumin was limited because of its low oral bioavailability, poor absorption, rapid metabolism, and elimination (Suresh et al. 2018). Nanomedicine, allow the formulation of therapeutic materials in a size of 1-1000 nm, which thoroughly correspond to the size of cells and their organelles (Villanueva-Flores et al. 2020). The formulation of curcumin in nanoparticles in a submicron size between 10 and $200 \mathrm{~nm}$, conserves the same properties of the phytochemical but with improved pharmacological effects due to its larger surface area, enhanced aqueous solubility, bioavailability, stability absorption, and cellular uptake (Arozal et al. 2019). In addition, curcumin has been approved as GRAS (Generally Recognized As Safe) by the United States Food and 
Drug Association and WHO Expert Committee on Food Additives (Gupta et al. 2013), besides, previous studies did not report apparent toxicity caused by oral intake of nanocurcumin (Ganugula et al. 2017).

We aim in the current study to investigate the potential effect of nanocurcumin to ameliorate mastitis in a rat model induced by LPS extracted from a pathogenic E.coli strain isolated from a bovine mastitis farm case in Alexandria governorate- Egypt.

\section{Materials And Methods \\ 2.1. Chemicals}

Nanocurcumin was purchased from NanoTech Egypt for photo-electronics (Al Giza, Egypt). MacConkey agar, eosin methylene agar, and blood agar base were purchased from Oxoid (Basingstoke, Hampshire, England). E. coli antisera were purchased from Denka Seiken (Tokyo, Japan). Rat sandwich enzymelinked immunosorbent assay (ELISA) Kits for Nrf2, TNF- $\alpha$, and IL-1 $\beta$ were purchased from Chongqing Biospes (China).

\subsection{Clinical examination of mastitis}

The clinical mastitis cases were diagnosed based on history, systemic signs including general conditions, rectal temperature, and appetite. Physical examination of udder for swelling, hotness, and pain. Milk appearance including color modification (yellow or tinged with blood), watery consistency, and presence of pus flakes. Then Milk samples were collected under strict hygienic conditions and each one was subjected to the California mastitis test (Schalm 1957), and the positive cases were subjected to bacteriological examination for isolation of E.coli.

\subsection{Bacteriological identification of E.coli and extraction of lipopolysaccharides}

\subsubsection{Isolation and identification of E.coli}

Each sample was thoroughly mixed, inoculated into Tripticase soya broth, incubated aerobically at $37^{\circ} \mathrm{C}$ overnight. Then, a loopful of inoculum from the broth culture was streaked onto $5 \%$ sheep blood, eosin Methylin agar, and MacConkey agar plates and incubated aerobically at $37^{\circ} \mathrm{C}$ for $24-48$ hours. Growth was observed daily and E.coli was identified according to its colonial characteristics including the colony size, shape, surface texture, color, consistency, the formation of a hemolysis on the blood agar (Supplementary Fig. 1); morphological shape as gram-negative bacilli after Gram staining and microscopical examination of the stained films. Then biochemical identification for the isolated E.coli colonies was performed according to (Quinn 1994) (Supplementary Table 1).

\subsubsection{Serological identification of E.coli}

Serotyping of E.coli isolates was performed according to (Edwards \&Ewing 1972) using 8 polyvalent and 43 monovalent $\mathrm{O}$ antisera and 22 monovalent $\mathrm{H}$ antisera for isolated E.coli. Macroscopic agglutination is 
only observed with the corresponding serotype following an antigen-antibody reaction by slide agglutination method test using a ready-made international trading diagnostic serum. The isolated E.coli strain from which LPS was extracted is $026: \mathrm{H} 4$.

\subsubsection{Extraction of lipopolysaccharides}

The methanol-chloroform method was used to extract LPS using the previously isolated and identified E.coli from a field bovine mastitis case. Suspension cultures were centrifuged for $30 \mathrm{~min}$ at 2500 to 3000 rpm, then the sediment was washed for 3 successive times by adding $2 \mathrm{ml}$ of ethanol 95\%, vortexed thoroughly, and centrifuged for $10 \mathrm{~min}$. The sediment was dried by placing the tube under a hood till the rest of the alcohol was evaporated, followed by the addition of $1 \mathrm{ml}$ EDTA $10 \%$, sonication, the addition of $1 \mathrm{ml}$ of saturated methanol/chloroform (1: 2 ratio), shaken for $2 \mathrm{~h}$, and centrifugation for $10 \mathrm{~min}$. Three layers were formed; methanol, left biomass including cell lysate, and chloroform top to button respectively. Chloroform and methanol layers were separated and transferred into new tubes and permit to evaporate completely, while the left biomass was discarded. Finally, the dried pellet was LPS (Mirzaei et al. 2011).

\subsubsection{Pilot test}

The pilot test had been done to ensure the correct and accurate effective dose of the prepared LPS for inducing the inflammatory condition to be used and to investigate the protocol that should be followed in the current study. Eight, lactating female albino rats that weighed $150 \pm 20 \mathrm{~g}$ were used, randomly allocated into four groups ( 2 rats for each group). The first group received a normal diet without treatment, while the 3 other groups were anesthetized and injected with 10, 20, $30 \mu \mathrm{g}$ LPS dissolved in $100 \mu \mathrm{l}$ sterile physiological saline into the teat canal of the inguinal mammary glands successively. After $24 \mathrm{~h}$, rats were sacrificed, and mammary samples were removed for histopathological examination (Chen et al. 2015, Ruifeng et al. 2014). According to the pilot test result, the accurate symptomatic LPS-induced mastitis dose was investigated as 30ug (Supplementary Fig. 2).

\subsection{Nanocurcumin characterization}

Nanocurcumin characterization was performed by transmission electron micrograph (TEM). Scanning of the aqueous dispersion denoted the particles are spherical-like shape and measures $50 \pm 5.5 \mathrm{~nm}$ (Fig. 1).

\subsection{Animals and experimental design}

Twenty adult white Wistar Albino female rats weighing $150 \pm 20 \mathrm{~g}$ were purchased from Animal Breeding Unit, Medical Research Institute, Alexandria University. The handling of rats were performed according to international ethical guidelines for the care and use of laboratory animals and the approval of experimental procedures were done by the Experimental Animal Use and Ethics Committee at the Faculty of Veterinary Medicine, Alexandria University, Egypt. The rats were housed in separate cages with a female-to-male ratio of 2:1 for breeding. They were provided ad libitum feed and water. Rats were kept at constant environmental and nutritional conditions in a well-ventilated room under a 12/12 hours light/dark cycle throughout the experiment. The animals were left two weeks for acclimatization before 
the beginning of the experiment. The lactating female rats were randomly allocated into four groups (5 rats each), classified as follows (considering the day of parturition is the zero-day): Group I was kept as healthy control, which was given no intramammary infusion. In Group II (LPS group), rats were given intramammary infusion with $30 \mu \mathrm{g}$ LPS dissolved in $100 \mu \mathrm{l}$ physiological saline on the 15th day of parturition following the method described by (Brouillette et al. 2005). Group III (nanocurcumin group) was given 7 doses of nanocurcumin $(35 \mathrm{mg} / \mathrm{kg} \mathrm{bw}$ ) dissolved in water once orally day after day by oral gavage from day 1 to day 14 post parturition. Group IV (prevention group) was pretreated from day 2 to day 14 after parturition with nanocurcumin $(35 \mathrm{mg} / \mathrm{kg} \mathrm{bw})$, On the 15th day after parturition, the offsprings were removed, then after $1 \mathrm{~h}$, the dams were infused with LPS. Twenty-four $\mathrm{h}$ after intramammary inoculation, the rats were anesthetized using ketamine/xylazine $(7.5-10 \mathrm{mg} / \mathrm{kg}, 1 \mathrm{mg} / \mathrm{kg}$ i.p). Rats were dissected to expose the MGs, and the fourth pair was collected and stored at $-80^{\circ} \mathrm{C}$ for further biochemical and molecular examination, while a presentative part of the MG tissue was excised and fixed in $10 \%$ formalin for later histopathological examination.

\subsection{Lipid peroxidation and antioxidant profile}

Malondialdehyde was determined according to the method described by (Draper \&Hadley 1990), total glutathione and GSSG content by the enzymatic method described by (Griffith 1980) in whole MG tissue homogenate.

\subsection{ELISA assays}

Nrf2, TNF- $\alpha$, and IL-1 $\beta$ levels in the mammary tissues were determined using ELISA kits for quantitative measurement of each parameter according to the instructions recommended by the manufacturer.

\subsection{RNA extraction and $q R T-P C R$}

Quantitative PCR was applied to determine the relative expression of TLR4, NF-KB p65, and HMGB1 using the specific primer sets for each gene (Table 1). About $30 \mathrm{mg} \mathrm{MG}$ tissue were rinsed in sterilized phosphate buffer saline and homogenized in liquid nitrogen using Teflon and pestle homogenizer then the homogenates were stored at $-80^{\circ} \mathrm{C}$ till RNA isolation. Total RNA was isolated using the RNeasy Mini Kit (Qiagen $\mathrm{GmbH}$, Germany) according to the manufacturer instructions. cDNA was synthesized from the purified RNA using QuantiTect Reverse Transcription Kit (Qiagen). The reaction mixture included RNA and master mix were placed at $42^{\circ} \mathrm{C}$ then inactivated at $95^{\circ} \mathrm{C}$. The qRT-PCR for the target genes was performed using QuantiTect SYBR Green PCR Master Mix (Qiagen Rotor-Gene Q). The fold change of mRNA expression was calculated after recording the $\mathrm{Ct}$ values for reference and target genes using the $2^{-\Delta \Delta C t}$ method. 
Table 1

Primer sequences used for qRT-PCR

\begin{tabular}{|c|c|c|c|}
\hline \multirow[t]{2}{*}{ Gene } & & \multirow[t]{2}{*}{ Sequence $\left(5^{\prime}-3^{\prime}\right)$} & \multirow{2}{*}{$\begin{array}{l}\text { GenBank } \\
\text { Accession No. }\end{array}$} \\
\hline & & & \\
\hline \multirow[t]{2}{*}{$T L R 4$} & $\mathrm{~F}:$ & CAGCAAAGTCCCTGATGACA & NM_019178.2 \\
\hline & $\mathrm{R}:$ & AGAGGTGGTGTAAGCCATGC & \\
\hline \multirow[t]{2}{*}{ NFKbp65 } & $\mathrm{F}:$ & CAGGACCAGGAACAGTTCGAA & NM_199267.2 \\
\hline & $\mathrm{R}$ : & CCAGGTTCTGGAAGCTATGGAT & \\
\hline \multirow[t]{2}{*}{ HMGB1 } & $\mathrm{F}:$ & TGATTAATGAATGAGTTCGGGC & NM_012963.2 \\
\hline & $\mathrm{R}:$ & TGCTCAGGAAACTTGACTGTTT & \\
\hline \multirow[t]{2}{*}{$G A P D H^{*}$} & $\mathrm{~F}:$ & GGGTGTGAACCACGAGAAATA & NM_017008.4 \\
\hline & $\mathrm{R}$ : & AGTTGTCATGGATGACCTTGG & \\
\hline * Houseke & Yor & & \\
\hline
\end{tabular}

\subsection{Histopathological examination}

The MG tissues for histopathological examination were fixed with $10 \%$ neutral buffered formalin for $24 \mathrm{~h}$. Tissue specimens were washed, dehydrated by serial dilutions of alcohol, cleared in xylene, and embedded in paraffin at $60^{\circ} \mathrm{C}$ in a hot air oven. Paraffin sections of 4-5 microns in thickness were prepared and stained with hematoxylin and eosin (HE) according to (Bancroft \&Gamble 2008) and examined under the light microscope.

\subsection{Statistical Analysis}

The obtained values are expressed as the mean \pm standard error (SE). Using the SPSS statistical package v22.0 for Windows (IBM, Armonk, NY, USA), one-way ANOVA followed by post hoc multiple comparisons Duncan's test were used to analyze obtained data. The significance level was set at $p \leq 0.05$. Kolmogorov-Smirnov's test has been used to test the normal distribution of data.

\section{Results}

\subsection{Effect of nanocurcumin on MG lipid peroxidation and glutathione redox in LPS-induced mastitis}

It is well known that the inflammatory process of MG is associated with accentuated lipid peroxidation and the most common way to examine the degree of lipid peroxidation is MDA level. Our results showed a significant upsurged MDA level in the MG of LPS stimulated group by approximately 3 folds above the 
control group. However, pre-treatment with oral administration of $35 \mathrm{mg} / \mathrm{kg}$ nanocurcumin dramatically declined the MDA level $(p<0.05)$ toward its level in the healthy group (Fig. 1A).

Intra-mammary infusion of LPS was associated with slightly decreased levels of total and reduced GSH and increased GSSG compared to the control group with markedly slumped GSH/GSSG ratio by nearly half that of the control group (Fig. 1B, C, D, E). Meanwhile, the nanocurcumin healthy group showed, an insignificant rise of nor total GSH and reduced GSH values or lower value of GSSG, however, GSH/GSSG ratio was moderately gone up in comparison to the control group. Among the pre-treated group, total and reduced GSH were moderately higher and the GSSG was lower than that of the LPS group. GSH/GSSG ratio denoted the same trend of reduced GSH; in particular a steady growth in the prevention group $(\mathrm{p}<$ 0.05) compared with that of the LPS group. Thus nanocurcumin effectively reversed the LPS inducedoxidative stress in the mammary tissue.

\subsection{Effect of nanocurcumin on MG Nrf2 in LPS-induced mastitis}

Nrf2, a redox-sensitive transcription factor, is important for cellular protection against oxidative stress through transcriptional upregulation of antioxidant proteins. LPS-induced mastitis in rats considerably decreased Nrf2 level in the MG tissue compared to the control group. On the contrary, Nrf2 significantly leveled up $(p<0.05)$ consequently to the oral administration of nanocurcumin either prior or following the induction of the inflammatory response as well as in healthy nanocurcumin group (Fig. 2.A)

\subsection{Effects of nanocurcumin on MG pro-inflammatory cytokines (TNF- $\alpha$ and IL-1 $\beta$ )}

Both TNF- $\alpha$ and IL-1 $\beta$ experienced rocketed-up trends following LPS inoculation above that of the control group, on the contrary, they encountered a noticeable downward drift in nanocurcumin prophylactic group $(p<0.05)$. Moreover, the nanocurcumin healthy group expressed small but significantly low proinflammatory cytokines values in comparison to the control group (Fig. 2b, c).

\subsection{Effects of nanocurcumin on the gene expression pattern of TLR4, NF-KB P65, and HMGB1 in LPS- induced mastitis}

The results of the MG tissue expressions of TLR4, NF-KB p65, and HMGB1 are presented in Fig. 3. On one hand, LPS challenge resulted in marked up-regulation of MG TLR4, NF-KB p65, and HMGB1 by around 6, 3.5 , and 2 folds respectively, in comparison to the control group. Nonetheless, the administration of nanocurcumin as a prophylactic against LPS-induced mastitis significantly suppressed the initiated expression of TLR4, NF-KB p65, and HMGB1.

\subsection{Effects of nanocurcumin on histopathological changes in LPS-induced rat mastitis}


Light microscopy examination of MG histopathological sections of control and nanocurcumin groups revealed the presence of normal architecture of alveoli which present with intact lining glandular epithelium and the presence of milk secretion remainant inside their lumen (Fig. 4a, b). On the other hand, histopathological examination of LPS group mammary glands revealed the presence of degeneration and desquamation of glandular epithelium of alveoli (Fig. 4c), focal interlobular mononuclear inflammatory cells infiltration (Fig. 4d, e) along with alveolar necrosis (Fig. 4f).

In contrast, the mammary glands of prevention group examination revealed also the presence of some degenerated and desquamated glandular epithelium within the ductile and alveolar lumen, however, most of the alveoli appeared with normal architecture along a wide area of the majority of the lobules (Fig. $4 \mathrm{~g}$, h).

\section{Discussion}

Mastitis is the most common and expensive development disease in dairy cows. Lower milk yield, lower milk quality, culling of chronically infected cows, and high veterinary care costs are the most significant economic consequences of mastitis. E. coli is the most frequently isolated coliform species from mastitis cases, likewise, it constitutes a crucial public health hazard (Fahim et al. 2019b). Since it is serious, often lethal, and difficult to manage with antibiotic injection, coliform mastitis caused by pathogenic E.coli is one of the most epidemic, frequent, and economically damaging diseases affecting dairy cattle worldwide (Seegers et al. 2003). Bacterial LPS, is the main component of the outer membrane of pathogenic gram-negative bacteria, induces acute inflammatory response initiated by its interaction with TLR4 resulting in the release of early (e.g., TNF-a) and "late" (e.g., HMGB1) pro-inflammatory cytokines. LPS commonly attacks the MG either exogenously via intramammary environmental inoculation, or as a diet-induced endogenous source, where LPS is released from the rumen (Sun et al. 2020).

Nowadays, there is a trend to intensify research concerning phytochemicals for their broad-spectrum benefits, making them a promising alternative for chemical therapeutics (Catanzaro et al. 2018). Curcumin is a major polyphenol with a variety of pharmacological and biological activities, despite its low bioavailability with a short half-life accounting for about 45 minutes with a minimal therapeutic effect (Eskiler et al. 2019), which has been recently tackled by nanoformulation of curcumin, which resulted in the elevation of its bioavailability and stability, and subsequently enhanced its immunomodulatory actions (Trivedi et al. 2017) as well as its intracellular accumulation (Ganugula et al. 2017). Our study illustrated, the capability of nanocurcumin to ameliorate mastitis induced by LPS extracted from E.coli isolated from a mastitic lactating cow case in Alexandria governorate- Egypt when used as a prohylaxis in rat model.

The current study revealed, that MDA has been substantially leveled up in MG tissue in response to the LPS challenge. Meanwhile, pre-oral administration of nanocurcumin had markedly declined it (Fig. 1A). It is known that LPS inoculation results in a dramatic increment of polymorphonuclear leukocytes (PMN) infiltration into the lumen of milk alveoli, (Spitzer et al. 2020), and generates ROS which is associated 
with lipid peroxidation resulting in oxidative damage of polyunsaturated fatty acids making them more susceptible to oxidation (Carvalho-Sombra et al.), generating a genotoxic breakdown by-product known as MDA (Colagar et al. 2009) which could inhibit the anti-oxidant molecules, inducing oxidative stress. In the contrast, nanocurcumin represses the formation of MDA regarding the efficient property of curcumin to scavenge lipid peroxide radicals such as ROS, superoxide radicals nitric oxide radicals, and hydrogen peroxide; due to its electron-donating phenolic hydroxyl group. Thus it inhibits the oxidation of thiol groups and prevents its deprivation, which is a fundamental step for cytoprotection (Panzarini et al. 2021).

Admittedly, GSH is the fundamental intracellular anti-oxidant as it scavenges ROS directly, besides, act indirectly as a cofactor that supports the enzymatic anti-oxidant activity. Under physiological status, GSH is present intracellularly in reduced form with only a few GSSG, which can be reverted to GSH by glutathione reductase. Therefore, the ratio between GSH and GSSG concentration represents a marker for oxidative stress due to the alteration of the GSH negative feedback loop (Bajic et al. 2019). The achieved data illustrated in Fig. 1 revealed that LPS stimulated MG tissue showed, decreased total GSH level which has been illustrated by a significantly increased GSH/GSSG ratio in comparison to the healthy rats, which is consequent to a higher GSSG level above the reduced GSH, and which are reversed under the influence of nanocurcumin. The production of reactive species is generally mediated by several pathways including the peroxisomal oxidation of fatty acids, and oxidative bursts in immune cells (Spitzer et al. 2020). As result, oxidative stress leads to macromolecular damages including proteins, lipids, and DNA, particularly, mitochondrial DNA. Hence, the diminished total GSH level contemplates a raised GSH oxidation by either reduction of $\mathrm{H}_{2} \mathrm{O}_{2}$ or other oxidants which is associated with increased GSSG and subsequently a steeply plunged GSH/GSSG ratio. Consequently, the declined anti-oxidant enzymes, and lipid peroxidation contributes to MG tissue damage during mastitis (Chinchali \&Kaliwal 2014).

Furthermore, Nrf2 has been identified as a key regulator transcription factor for anti-oxidant signaling (Zangui et al. 2019). Under homeostatic conditions, it is kept inactive in the cytoplasm bound to Keap1. In response to oxidants, Keap1 becomes oxidized leading to release, and nuclear translocation of Nrf2, where it binds to ARE on the DNA to induce the transcription of both antioxidant and phase II detoxification enzymes including SOD, GPx, CAT, HOX1, GT, GR, and NQ01 resulting in the preservation of the redox potential of the mitochondria (Fusco et al. 2020). On the contrary, nanocurcumin possesses an enhanced anti-oxidant nature owing to its expanded surface area offering it the opportunity to expose more hydroxyl groups (Moghaddasi et al. 2018), preventing the generation of superoxides and hydrogen peroxide (Aggarwal et al. 2013). Moreover, the a, $\beta$ unsaturated ketone groups bind covalently to the cysteine residue of Keap1, activating the Keap1/ Nrf2/ ARE system (Ji et al. 2020), leading to the release and nuclear translocation of Nrf2.

Another factor to consider, the lipid A fraction of LPS which recognized by LBP and CD14 which transferred the LPS molecule to MD2 /TLR4, forming a ternary complex after dimerization at a picomolar concentration (Weiss \&Barker 2018), provoking the initiation of inflammatory response involving the activation and recruitment of PMN into the MG tissue, release of cytokines through the stimulation of 
intra-mammary innate immune response resulting in tissue damage (Bhattarai et al. 2018, Weiss \&Barker 2018), thus TLR4 is considered as a key regulator for mastitis (He et al. 2015). The procured results from Figs. 2 and 3 illustrated the inflammatory response during LPS-induced mastitis in rats by dramatically climbed relative expression of TLR4, NF-KB, and HMGB1 together with TNF- $\alpha$ and IL-1 $\beta$ levels. This could be explained by the fact of the activation of TLR4 downstream signaling by LPS (Wang et al. 2015), including the combination of TLR domain of TIRAP with TIR domain of TLR4, followed by the recruitment of MYD88, then the activation of IKK (Li et al. 2020)which catalyzes the phosphorylation of IKBa, followed by its proteasomal degradation, and consecutively the separation and nuclear translocation of NF-KB p65 (Panaro et al. 2020) where it binds to DNA and triggers the expression of genes encoding inflammatory cytokines including IL-1 $\beta$, TNF- $\alpha$, and IL-6 (Fu et al. 2013, Fu et al. 2014).

HMGB1 is expressed in all eukaryotic cells as an advanced, greatly conserved, and abundant nuclear protein; during normal healthy conditions, it bounds to DNA in order to maintain the stabilization as well as regulate gene transcription; nevertheless, during pathological status, it serves as a DAMP after being released extracellularly either actively succeeding cellular stimulation by LPS or cytokines; or passively from damaged cells (Andersson et al. 2018, Elfeky et al. 2018, Wang et al. 2015). In response to an inflammatory process, it becomes upregulated, to perform as a novel endogenous ligand for TLR4/MD2 complex, RAGE and TLR2 to aggravate the action of LPS via triggering the upregulation of MYD88, resulting in further expression of pro-inflammatory cytokines, NF-KB and TLR4. Furthermore, HMGB1 could bind to the inactive pro-IL-1 $\beta$ forming HMGB1/pro-IL-1 $\beta$ complex, inducing the transcription of other inflammatory cytokines. Thus, HMGB1 has been identified to play a key role in the regulation of chronic inflammatory response (Prantner et al. 2020, Yang et al. 2018).

Therefore, the suppression of NF-KB and HMGB1 activities is thought to be an effective approach to alleviate the inflammatory response during mastitis (Sun et al. 2020, Wang et al. 2012). Our results including the significant downregulation of the inflammatory indices in the MG tissue of LPS challenged experimental animals, under the influence of orally administrated nanocurcumin, clarified this hypothesis. Nanocurcumin reduces the number of PMN recruited to the site of inflammation (Zambrano et al. 2018). It antagonizes TLR4/MYD88/NF-kB cascade (Meng et al. 2018) at disparate points. For instance, the a, $\beta$ unsaturated 1,3 diketone moiety of curcumin allow it to bind non covalently with MD2, preventing the recognition and interaction between LPS and TLR4 and prohibit the dimerization of the receptor complex and its downstream signaling (Panaro et al. 2020) (Hassanzadeh et al. 2020). Furthermore, curcumin interfered with TRAF6, potentially inhibited IKK, the phosphorylation of IKBa, and maintained the cytoplasmic inactive form of NF-KB (Fallahi et al. 2021, Panaro et al. 2020). Moreover, curcumin suppressed the gene expression of TLR4, MYD88 (Hassanzadeh et al. 2020), NF-KB, as well as IL-1 $\beta$, TNF- $\alpha$ (Bisht et al. 2020), and HMGB1 (Da et al. 2019); Besides, curcumin inhibits HMGB1 induced signaling by both suppressing its extra-nuclear translocation, and hampering de novo synthesized HMGB1 (Wang et al. 2012). Moreover, the cytoprotective impact of curcumin against HMGB1 has been designated by the potential of curcumin to downregulating TLR4 and TLR2 expression (Kim et al. 2011). Lastly, (Suresh et al. 2018) attested that nanocurcumin pre-treatment significantly down-regulated the 
expression of NF-kB P65 and diminished the level of inflammatory cytokines in Staph. aureus- induced mastitis in laboratory animal model.

\section{Conclusion}

To sum up, our survey designated that prophylactic oral intake of nanocurcumin exhibited anti-oxidant and anti-inflammatory impact against LPS-induced rat mastitis model, which may be related to the amelioration of redox status of the mammary gland tissue through the activation of Nrf2, preservation of GSH pool and the suppression of lipid peroxidation, as well as the downregulation of key inflammatory modulators including TLR4, NF-KB p65, and HMGB1, besides the suppression of pro-inflammatory cytokines production, as well as the amelioration of the histopathological structure of the mammary gland. Overall, our findings suggest that nanocurcumin feed supplement may be a promising approach for further implementation in mastitis control measures.

\section{Declarations}

Availability of data and materials

All data analyzed during this study are included in this article.

Author contributions

Ingi H. Elmassry: Funding acquisition, Investigation, and. Nabil M. Taha: Conceptualization, Investigation, and. Mohamed A. Lebda: Conceptualization, Investigation, Writing - original draft Writing - original draft, submission and follow up the peer-review process,.and. Mohamed S. Elfeky: Conceptualization, Investigation, and Formal analysis.

Funding

Not applicable

Consent to publish

All authors have read and approved the final version of the manuscript for publication.

Competing interests

The authors declare that they have no competing interests.

Ethics approval and consent to participate

All authors carefully read and approved the study.

\section{References}


Aggarwal BB, Gupta SC, Sung BJBjop (2013): Curcumin: an orally bioavailable blocker of TNF and other pro-inflammatory biomarkers. 169, 1672-1692

Andersson U, Yang H, Harris H (2018): Extracellular HMGB1 as a therapeutic target in inflammatory diseases. Expert opinion on therapeutic targets 22, 263-277

Arozal W, Ramadanty WT, Louisa M, Satyana RPU, Hartono G, Fatrin S, Purbadi S, Estuningtyas A, Instiaty IJDr (2019): Pharmacokinetic profile of curcumin and nanocurcumin in plasma, ovary, and other tissues. $69,559-564$

Bajic VP, Van Neste C, Obradovic M, Zafirovic S, Radak D, Bajic VB, Essack M, Isenovic ERJOm, longevity c (2019): Glutathione "redox homeostasis" and its relation to cardiovascular disease. 2019

Bancroft JD, Gamble M (2008): Theory and practice of histological techniques. Elsevier health sciences

Barreto F, Jank L, Castilhos T, Rau RB, Tomaszewski CA, Ribeiro C, Hillesheim DR (2019): Chemical residues and mycotoxins in raw milk, Raw milk. Elsevier, pp. 273-293

Bhattarai D, Worku T, Dad R, Rehman ZU, Gong X, Zhang SJMp (2018): Mechanism of pattern recognition receptors (PRRs) and host pathogen interplay in bovine mastitis. 120, 64-70

Bisht A, Dickens M, Rutherfurd-Markwick K, Thota R, Mutukumira AN, Singh HJN (2020): Chlorogenic Acid Potentiates the Anti-Inflammatory Activity of Curcumin in LPS-Stimulated THP-1 Cells. 12, 2706

Boyanapalli SS, Paredes-Gonzalez X, Fuentes F, Zhang C, Guo Y, Pung D, Saw CLL, Kong A-NTJCRiT (2014): Nrf2 knockout attenuates the anti-inflammatory effects of phenethyl isothiocyanate and curcumin. 27, 2036-2043

Brouillette E, Malouin FJM, infection (2005): The pathogenesis and control of Staphylococcus aureusinduced mastitis: study models in the mouse. 7, 560-568

Carvalho-Sombra T, Fernandes D, Bezerra B, Nunes-Pinheiro DJV, Science A Systemic inflammatory biomarkers and somatic cell count in dairy cows with subclinical mastitis. 100165

Catanzaro M, Corsini E, Rosini M, Racchi M, Lanni CJM (2018): Immunomodulators inspired by nature: a review on curcumin and echinacea. 23, 2778

Chen C-Y, Kao C-L, Liu C-MJljoms (2018): The cancer prevention, anti-inflammatory and anti-oxidation of bioactive phytochemicals targeting the TLR4 signaling pathway. 19, 2729

Chen J, Xu J, Li J, Du L, Chen T, Liu P, Peng S, Wang M, Song HJli (2015): Epigallocatechin-3-gallate attenuates lipopolysaccharide-induced mastitis in rats via suppressing MAPK mediated inflammatory responses and oxidative stress. $26,147-152$ 
Cheng WN, Han SGJA-AJoAS (2020): Bovine mastitis: risk factors, therapeutic strategies, and alternative treatments - A review. 33,1699

Chinchali JF, Kaliwal BBJI-AJPR (2014): EFFECT OF MASTITIS ON MAMMARY GLAND BIOCHEMICAL AND OXIDATIVE STRESS PARAMETERS IN EXPERIMENTALLY INDUCED LACTATING MICE. 4, 1607-1614

Cobirka M, Tancin V, Slama P (2020): Epidemiology and Classification of Mastitis. 10, 2212

Colagar AH, Pouramir M, Marzony ET, Jorsaraei SGAJBAoB, Technology (2009): Relationship between seminal malondialdehyde levels and sperm quality in fertile and infertile men. 52, 1387-1392

Da W, Zhang J, Zhang R, Zhu JJljoi, pharmacology (2019): Curcumin inhibits the lymphangiogenesis of gastric cancer cells by inhibiton of HMGB1/VEGF-D signaling. 33, 2058738419861600

de Almeida Alvarenga L, de Oliveira Leal V, Borges NA, de Aguiar AS, Faxén-Irving G, Stenvinkel P, Lindholm B, Mafra DJJoFF (2018): Curcumin-A promising nutritional strategy for chronic kidney disease patients. $40,715-721$

Draper HH, Hadley MJMie (1990): [43] Malondialdehyde determination as index of lipid Peroxidation. $186,421-431$

Edwards P, Ewing WJCMM (1972): Identification of Enterobacteriaceae, Burgess Publ. 103-104

Elfeky M, Yoneshiro T, Okamatsu-Ogura Y, Kimura K (2018): Adiponectin suppression of late inflammatory mediator, HMGB1-induced cytokine expression in RAW264 macrophage cells. Journal of biochemistry $163,143-153$

Eskiler GG, Özkan AD, Kaleli S, Bilir CJJoP, Sciences P (2019): Inhibition of TLR4/TRIF/IRF3 signaling pathway by curcumin in breast cancer cells. 22, 281-291

Fahim KM, Ismael E, Khalefa HS, Farag HS, Hamza DA (2019a): Isolation and characterization of E. coli strains causing intramammary infections from dairy animals and wild birds. International Journal of Veterinary Science and Medicine 7, 61-70

Fahim KM, Ismael E, Khalefa HS, Farag HS, Hamza DAJljovs, medicine (2019b): Isolation and characterization of E. coli strains causing intramammary infections from dairy animals and wild birds. 7 , $61-70$

Fallahi F, Borran S, Ashrafizadeh M, Zarrabi A, Pourhanifeh MH, Mahabady MK, Sahebkar A, Mirzaei HJMI (2021): Curcumin and inflammatory bowel diseases: From in vitro studies to clinical trials. $130,20-30$

Fu Y, Zhou E, Liu Z, Li F, Liang D, Liu B, Song X, Zhao F, Fen X, Li DJVi, immunopathology (2013): Staphylococcus aureus and Escherichia coli elicit different innate immune responses from bovine mammary epithelial cells. 155, 245-252 
Fu Y, Zhou E, Wei Z, Liang D, Wang W, Wang T, Guo M, Zhang N, Yang Z (2014): Glycyrrhizin inhibits the inflammatory response in mouse mammary epithelial cells and a mouse mastitis model. The FEBS journal 281, 2543-57

Fusco R, Cordaro M, Siracusa R, Peritore AF, D’Amico R, Licata P, Crupi R, Gugliandolo EJA (2020): Effects of Hydroxytyrosol against Lipopolysaccharide-Induced Inflammation and Oxidative Stress in Bovine Mammary Epithelial Cells: A Natural Therapeutic Tool for Bovine Mastitis. 9, 693

Ganugula R, Arora M, Jaisamut P, Wiwattanapatapee R, Jørgensen HG, Venkatpurwar VP, Zhou B, Rodrigues Hoffmann A, Basu R, Guo SJBjop (2017): Nano-curcumin safely prevents streptozotocininduced inflammation and apoptosis in pancreatic beta cells for effective management of Type 1 diabetes mellitus. 174, 2074-2084

Glynn DJ, Hutchinson MR, Ingman WVJBor (2014): Toll-like receptor 4 regulates lipopolysaccharideinduced inflammation and lactation insufficiency in a mouse model of mastitis. 90, 91, 1-11

Gomes F, Henriques M (2016): Control of Bovine Mastitis: Old and Recent Therapeutic Approaches. Current microbiology $72,377-82$

Griffith OWJAb (1980): Determination of glutathione and glutathione disulfide using glutathione reductase and 2-vinylpyridine. 106, 207-212

Gupta SC, Patchva S, Koh W, Aggarwal BBJC, pharmacology e, physiology (2012): Discovery of curcumin, a component of golden spice, and its miraculous biological activities. 39, 283-299

Gupta SC, Patchva S, Aggarwal BBJTAj (2013): Therapeutic roles of curcumin: lessons learned from clinical trials. $15,195-218$

Hassanzadeh S, Read MI, Bland AR, Majeed M, Jamialahmadi T, Sahebkar AJPr (2020): Curcumin: an inflammasome silencer. 104921

He X, Wei Z, Zhou E, Chen L, Kou J, Wang J, Yang ZJli (2015): Baicalein attenuates inflammatory responses by suppressing TLR4 mediated NF-KB and MAPK signaling pathways in LPS-induced mastitis in mice. $28,470-476$

Ingman WV, Glynn DJ, Hutchinson MRJJomgb, neoplasia (2014): Inflammatory mediators in mastitis and lactation insufficiency. 19, 161-167

Jang J, Hur HG, Sadowsky MJ, Byappanahalli M, Yan T, Ishii SJJoam (2017): Environmental Escherichia coli: ecology and public health implications-a review. 123, 570-581

Ji L, Qu L, Wang C, Peng W, Li S, Yang H, Luo H, Yin F, Lu D, Liu XJEJoMC (2020): Identification and optimization of piperlongumine analogues as potential antioxidant and anti-inflammatory agents via activation of Nrf2. 210, 112965

Page 15/23 
Jiang A, Zhang Y, Zhang X, Wu D, Liu Z, Li S, Liu X, Han Z, Wang C, Wang JJli (2020): Morin alleviates LPS-induced mastitis by inhibiting the PI3K/AKT, MAPK, NF-KB and NLRP3 signaling pathway and protecting the integrity of blood-milk barrier. 78, 105972

Kan X, Liu B, Guo W, Wei L, Lin Y, Guo Y, Gong Q, Li Y, Xu D, Cao YJJocp (2019): Myricetin relieves LPSinduced mastitis by inhibiting inflammatory response and repairing the blood-milk barrier. $234,16252-$ 16262

Khan MZ, Khan A, Xiao J, Ma J, Ma Y, Chen T, Shao D, Cao ZJA (2020): Overview of Research Development on the Role of NF-kB Signaling in Mastitis. 10, 1625

Kibebew KJJBAH (2017): Bovine mastitis: A review of causes and epidemiological point of view. 7, 1-14 Kim D-C, Lee W, Bae J-SJIR (2011): Vascular anti-inflammatory effects of curcumin on HMGB1-mediated responses in vitro. $60,1161-1168$

Li X, Zhong C-Q, Yin Z, Qi H, Xu F, He Q, Shuai JJljoms (2020): Data-Driven Modeling Identifies TIRAPIndependent MyD88 Activation Complex and Myddosome Assembly Strategy in LPS/TLR4 Signaling. 21, 3061

Mazgaeen L, Gurung PJljoms (2020): Recent advances in lipopolysaccharide recognition systems. 21, 379

Meng F-C, Zhou Y-Q, Ren D, Wang R, Wang C, Lin L-G, Zhang X-Q, Ye W-C, Zhang Q-WJN, agents af, dyes $f$ (2018): Turmeric: A review of its chemical composition, quality control, bioactivity, and pharmaceutical application. 299-350

Mirzaei A, Ashtiani MHHRA, Rahbar M, Rastegar HJSR, Essays (2011): A simple method for non phenolic extraction of lipopolysaccharide from Salmonella typhimurium and Salmonella enteritidis with high purity and pyrogenicity in rat. $6,1101-1105$

Moghaddasi F, Housaindokht MR, Darroudi M, Bozorgmehr MR, Sadeghi AJL (2018): Synthesis of nano curcumin using black pepper oil by O/W Nanoemulsion Technique and investigation of their biological activities. 92, 92-100

Panaro MA, Corrado A, Benameur T, Paolo CF, Cici D, Porro CJljoms (2020): The emerging role of curcumin in the modulation of TLR-4 signaling pathway: focus on neuroprotective and anti-rheumatic properties. 21, 2299

Panzarini E, Mariano S, Tacconi S, Carata E, Tata AM, Dini LJN (2021): Novel Therapeutic Delivery of Nanocurcumin in Central Nervous System Related Disorders. 11, 2

Prantner D, Nallar S, Vogel SNJTFJ (2020): The role of RAGE in host pathology and crosstalk between RAGE and TLR4 in innate immune signal transduction pathways. 34, 15659-15674 
Priyadarsini KIJM (2014): The chemistry of curcumin: from extraction to therapeutic agent. 19, 2009120112

Pu H-L, Chiang W-L, Maiti B, Liao Z-X, Ho Y-C, Shim MS, Chuang E-Y, Xia Y, Sung H-WJAn (2014): Nanoparticles with dual responses to oxidative stress and reduced $\mathrm{pH}$ for drug release and antiinflammatory applications. 8, 1213-1221

Quesnell R, Klaessig S, Watts J, Schukken YJJods (2012): Bovine intramammary Escherichia coli challenge infections in late gestation demonstrate a dominant antiinflammatory immunological response. 95, 117-126

Quinn PJ (1994): Clinical veterinary microbiology

Rahimi HR, Nedaeinia R, Shamloo AS, Nikdoust S, Oskuee RKJAjop (2016): Novel delivery system for natural products: Nano-curcumin formulations. 6,383

Rai M, Pandit R, Gaikwad S, Yadav A, Gade AJNR (2015): Potential applications of curcumin and curcumin nanoparticles: from traditional therapeutics to modern nanomedicine. 4, 161-172

Ruifeng G, Yunhe F, Zhengkai W, Yimeng L, Minjun Y, Xiaojing S, Zhengtao Y, Naisheng ZJEJoP (2014): Chlorogenic acid attenuates lipopolysaccharide-induced mice mastitis by suppressing TLR4-mediated NF-kB signaling pathway. 729, 54-58

Sampath V (2018): Bacterial endotoxin-lipopolysaccharide; structure, function and its role in immunity in vertebrates and invertebrates. Agriculture and Natural Resources 52, 115-120

Sayed H, Salama SS, Soliman TJGV (2014): Bacteriological evaluation of present situation of mastitis in dairy cows. $13,690-695$

Schalm OJJAVMA (1957): Experiments and observations leading to development of the California Mastitis Test. 130, 199-204

Seegers H, Fourichon C, Beaudeau FJVr (2003): Production effects related to mastitis and mastitis economics in dairy cattle herds. 34, 475-491

Shi H, Guo Y, Liu Y, Shi B, Guo X, Jin L, Yan SJAn (2016): The in vitro effect of lipopolysaccharide on proliferation, inflammatory factors and antioxidant enzyme activity in bovine mammary epithelial cells. 2, 99-104

Smulski S, Gehrke M, Libera K, Cieslak A, Huang H, Patra AK, Szumacher-Strabel M (2020): Effects of various mastitis treatments on the reproductive performance of cows. BMC veterinary research 16, 99

Spitzer AJ, Tian Q, Choudhary RK, Zhao F-QJOm, longevity c (2020): Bacterial endotoxin induces oxidative stress and reduces milk protein expression and hypoxia in the mouse mammary gland. 2020 
Sun X, Luo S, Jiang C, Tang Y, Cao Z, Jia H, Xu Q, Zhao C, Loor JJ, Xu CJJods (2020): Sodium butyrate reduces bovine mammary epithelial cell inflammatory responses induced by exogenous lipopolysaccharide, by inactivating NF-KB signaling. 103, 8388-8397

Suojala L, Kaartinen L, Pyörälä SJJovp, therapeutics (2013): Treatment for bovine E scherichia coli mastitis-an evidence-based approach. 36, 521-531

Suresh S, Sankar P, Telang AG, Kesavan M, Sarkar SNJli (2018): Nanocurcumin ameliorates Staphylococcus aureus-induced mastitis in mouse by suppressing NF-KB signaling and inflammation. $65,408-412$

Trivedi MK, Mondal SC, Gangwar M, Jana SJI (2017): Immunomodulatory potential of nanocurcuminbased formulation. 25, 609-619

Villanueva-Flores F, Castro-Lugo A, Ramírez OT, Palomares LA (2020): Understanding cellular interactions with nanomaterials: towards a rational design of medical nanodevices. Nanotechnology 31, 132002132002

Wang C, Nie H, Li K, Zhang Y-x, Yang F, Li C-b, Wang C-f, Gong QJEjop (2012): Curcumin inhibits HMGB1 releasing and attenuates concanavalin A-induced hepatitis in mice. 697, 152-157

Wang F-C, Pei J-X, Zhu J, Zhou N-J, Liu D-S, Xiong H-F, Liu X-Q, Lin D-J, Xie YJWJoGW (2015): Overexpression of HMGB1 A-box reduced lipopolysaccharide-induced intestinal inflammation via HMGB1/TLR4 signaling in vitro. 21,7764

Weiss J, Barker JJF (2018): Diverse pro-inflammatory endotoxin recognition systems of mammalian innate immunity. 7

Yang Y, Han C, Guo L, Guan QJB, behavior (2018): High expression of the HMGB1-TLR4 axis and its downstream signaling factors in patients with Parkinson's disease and the relationship of pathological staging. 8, e00948

Zambrano LM, Brandao DA, Rocha FR, Marsiglio RP, Longo IB, Primo FL, Tedesco AC, Guimaraes-Stabili MR, Junior CRJSr (2018): Local administration of curcumin-loaded nanoparticles effectively inhibits inflammation and bone resorption associated with experimental periodontal disease. 8, 1-11

Zangui M, Atkin SL, Majeed M, Sahebkar AJPr (2019): Current evidence and future perspectives for curcumin and its analogues as promising adjuncts to oxaliplatin: state-of-the-art. 141, 343-356

\section{Figures}



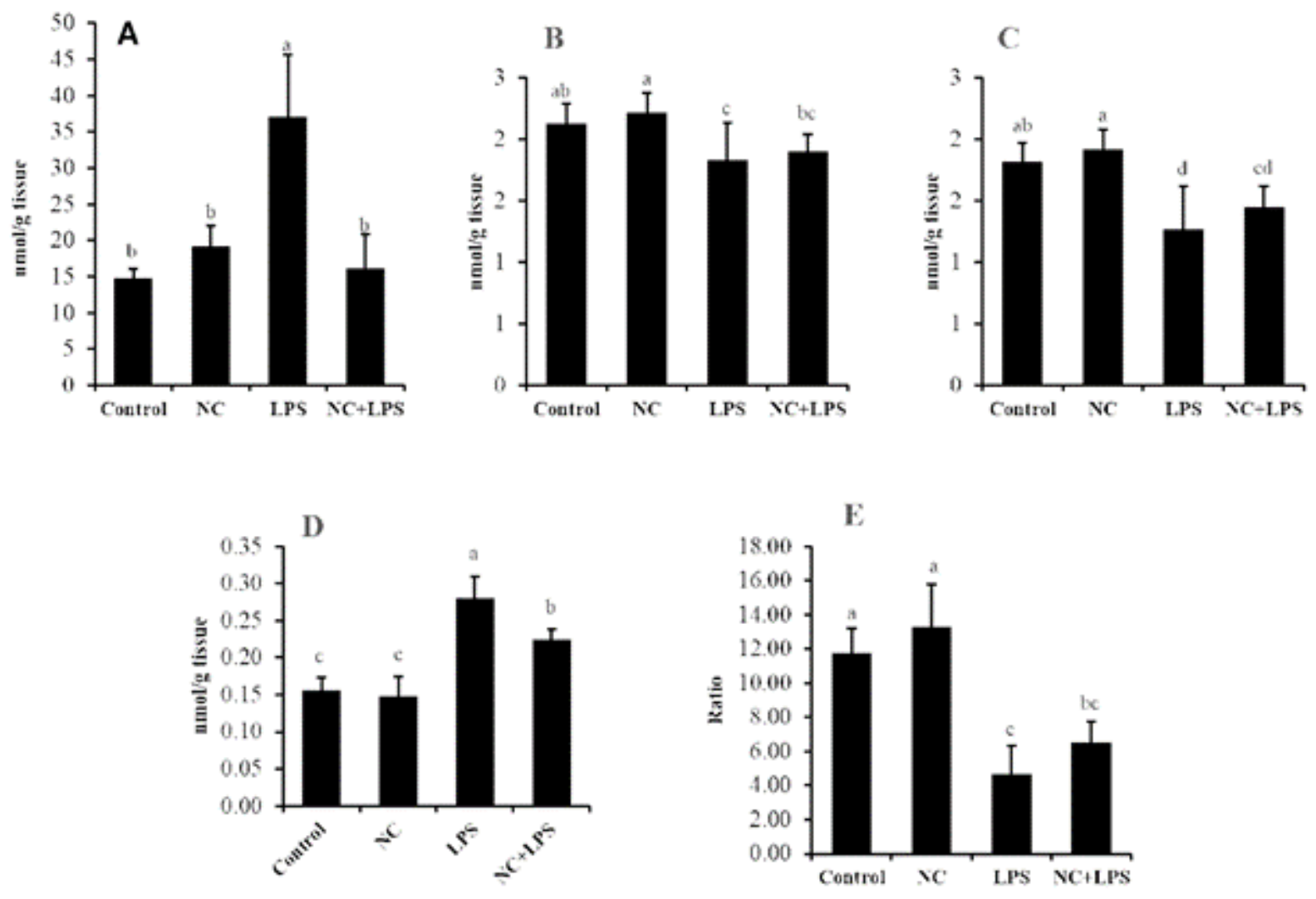

\section{Figure 1}

Effects of nanocurcumin on the lipid peroxidation biomarker and glutathione redox in rat mammary tissues. (A); MDA, (B); total GSH, (C); GSH, (D); GSSG, and €; GSH/GSSG ratio. The expressed values are the mean $\pm S E$. Means denoted with different superscripts are significantly $(P<0.05)$. 

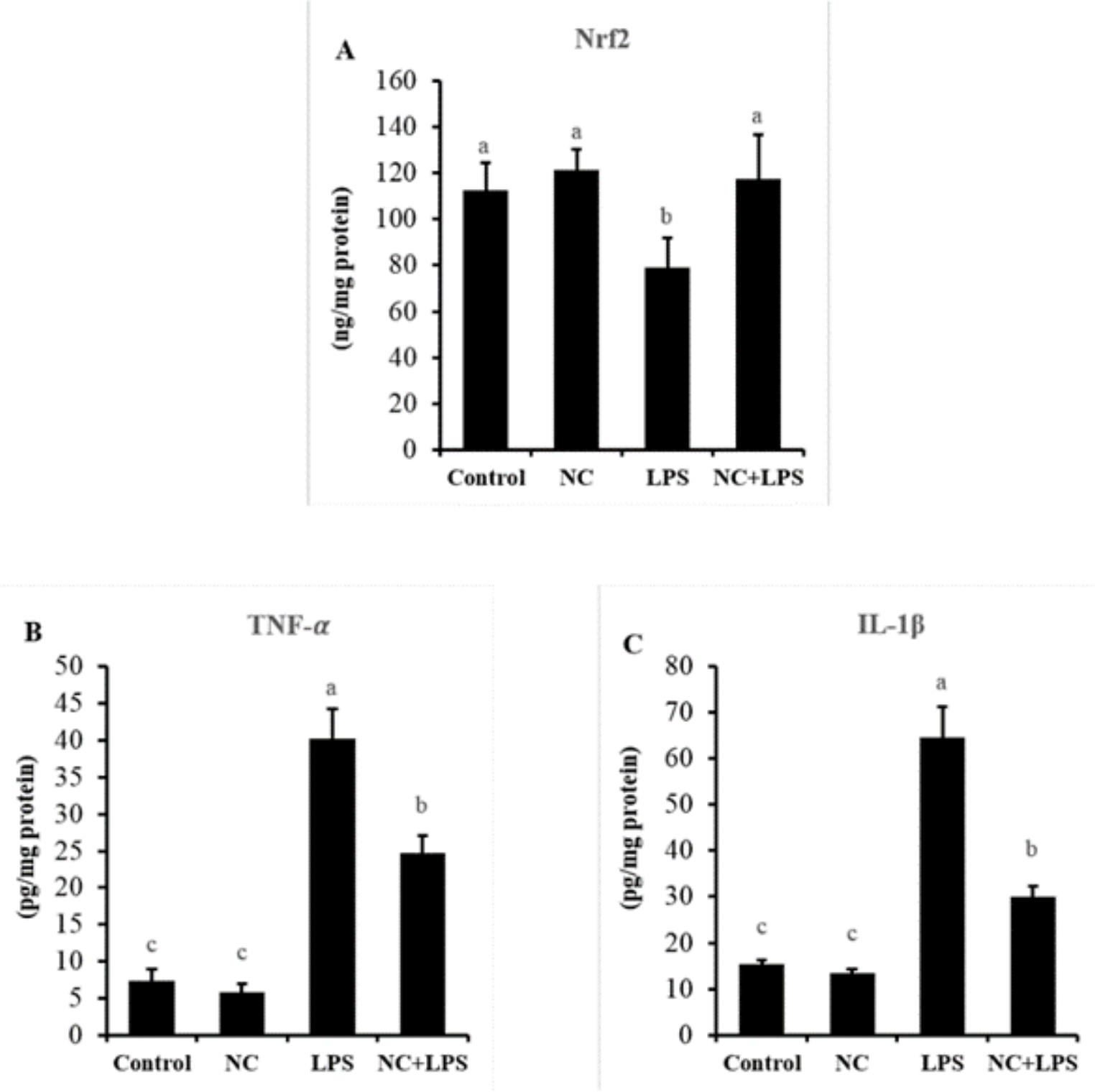

Figure 2

The levels of Nrf2 (A), TNF-a (B) and IL-1 3 (C) in rat mammary tissues. The expressed values are the mean $\pm S E$. Means denoted with different superscripts are significantly $(P<0.05)$. 

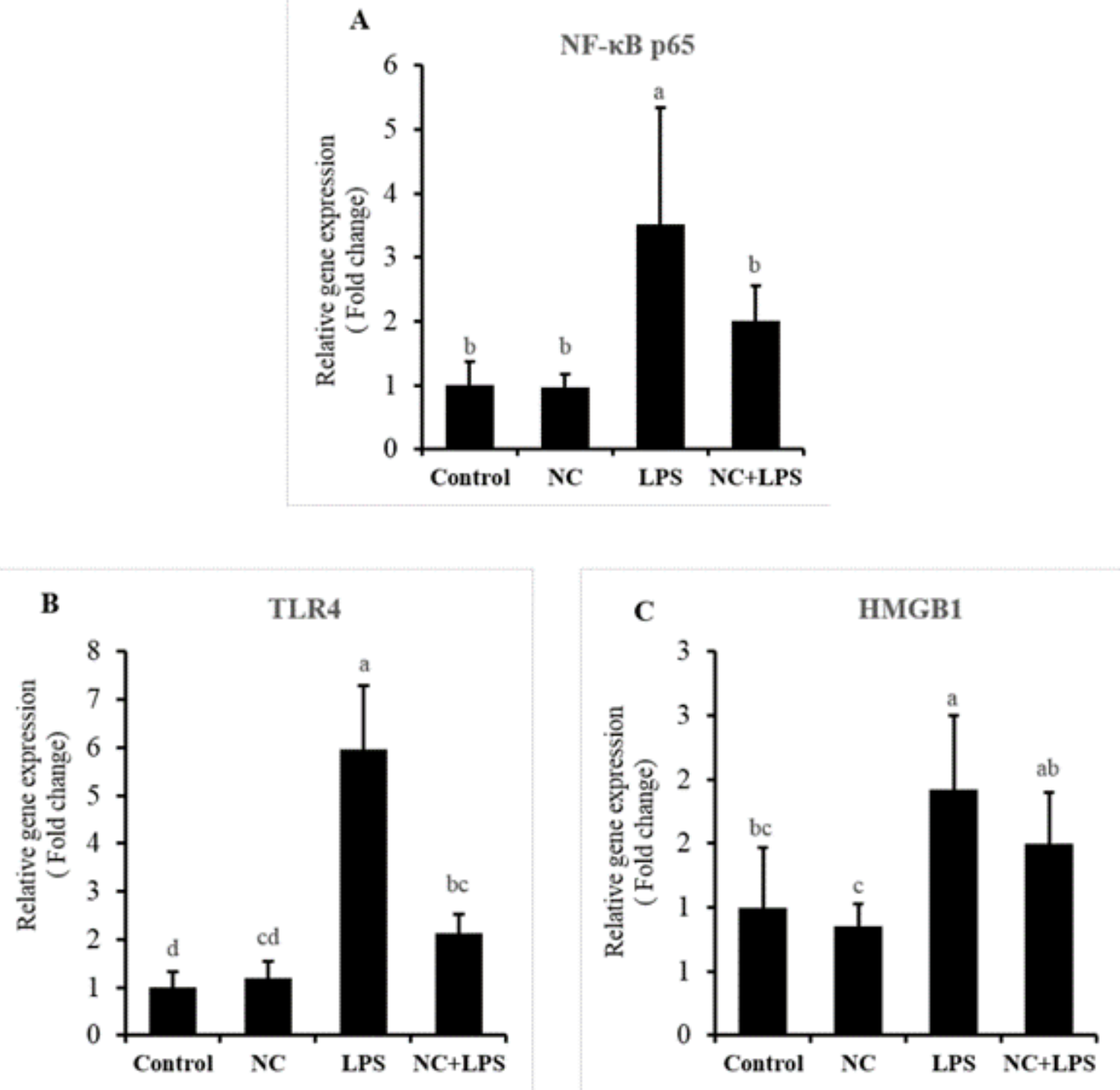

Figure 3

mRNA expression patterns of NF-KB p65 (A), TLR4 (B) and HMGB1 (C) in rat mammary tissues. The expressed values are the mean \pm SE. Means denoted with different superscripts are significantly $(P<0.05)$. 


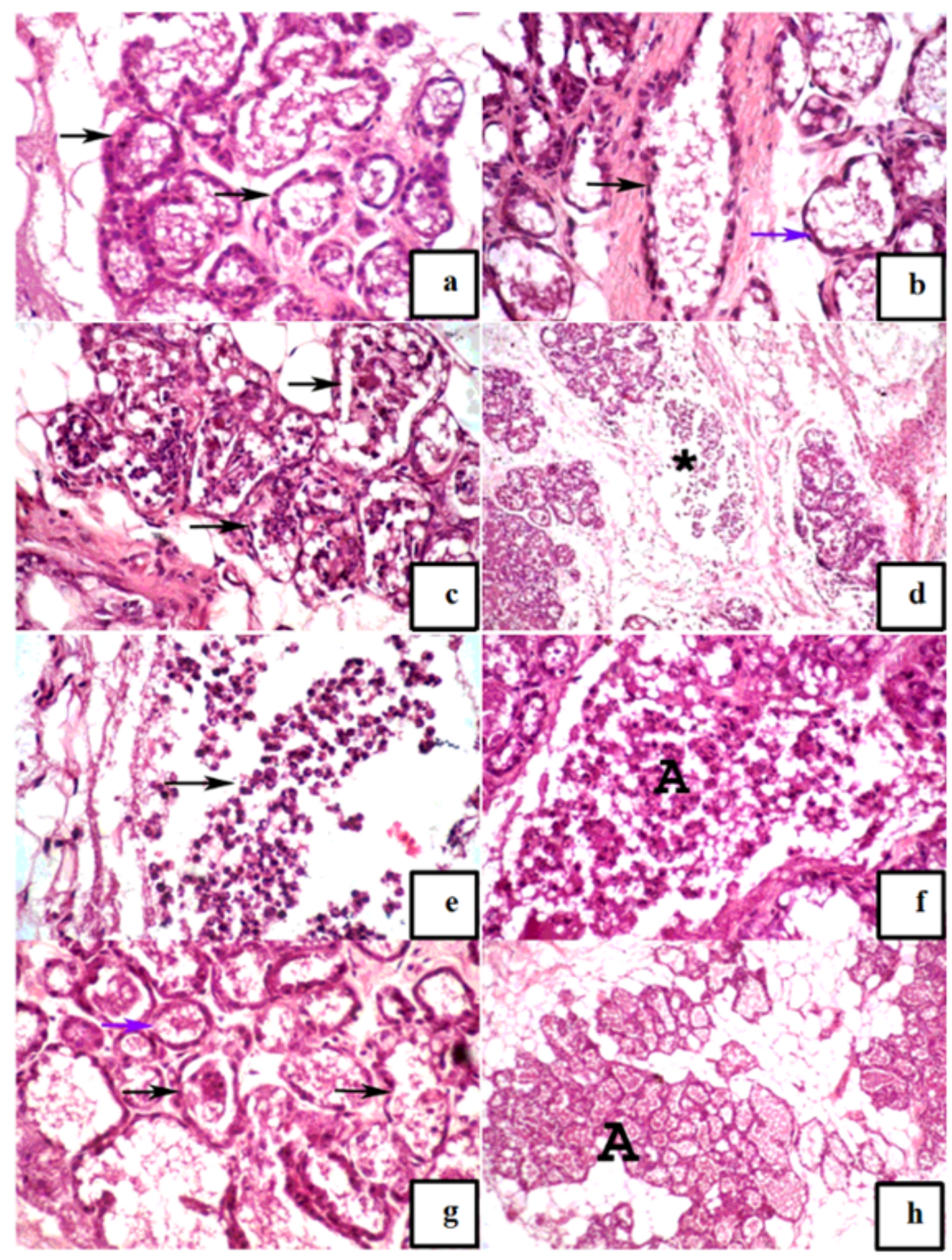

Figure 4

Photomicrograph of rat mammary tissues stained with HE (a) Control group MG showing the presence of normal architecture and details with the presence of normal intact glandular epithelium and presence of milk secretion remainant within the alveolar lumen (arrows), (×400). (b) Nanocurcumin group MG showing the presence of apparently normal alveoli with intact glandular epithelium (blue arrow) and duct (black arrow), (×400). LPS group MG showing; (c) degeneration of glandular lining epithelium of alveoli 
and its desquamation within the alveolar lumen (black arrow), $(\times 400)$, focal interlobular mononuclear inflammatory cells infiltration (asterisk), $(\times 200, d),(\times 400, e)$, and (f) focal alveolar necrosis with mononuclear inflammatory cells infiltration $(A),(\times 400)$. Prevention group MG showing; $(g)$ the presence of apparently normal alveoli with intact glandular epithelium (blue arrow) and presence of some alveoli with desquamated glandular epithelium within the lumen (black arrow), $(\times 400)$ and (h) the presence of apparently normal mammary lobule and alveoli with remainant milk secretion inside $(A),(\times 200)$.

\section{Supplementary Files}

This is a list of supplementary files associated with this preprint. Click to download.

- Supplementary.docx 